\title{
Feasibility of a three-dimensional rotational angiography road map for individualized guidance of uterine artery catheterization
}

\author{
W.Q. Li ${ }^{1, *}$, X.S. Deng ${ }^{1}$, G.B. Li ${ }^{2}$, Y.G. Kang ${ }^{2}$ \\ ${ }^{1}$ Department of Interventional Radiology, Affiliated Hospital of Hunan institute of traditional Chinese medicine, \\ Changsha 410013, Hunan (P.R. China) \\ ${ }^{2}$ Department of Interventional Radiology, Xiangtan Central Hospital, Xiangtan 411100, Hunan (P.R. China)
}

\begin{abstract}
Summary
Objectives: This study aimed to compare the feasibility and advantages of a three-dimensional rotational angiography (3DRA) road map for individualized guidance of uterine artery (UA) catheterization with those of a conventional two-dimensional (2D) road map. Materials and Methods: Twenty patients were randomly assigned to the 3DRA and 2D groups for UA catheterization and embolization for symptomatic uterine fibroid, postpartum haemorrhage, incision pregnancy, adenomyosis, and cervical cancer in the past 2 years at our hospital. Demographic data such as patient age, abdominal perimeter, and uterine diseases were recorded. Procedure time, fluoroscopy time, contrast medium volume, and patient radiation dose were also recorded. There was no difference between the two groups in terms of demographic data. Results: All 20 patients were successfully catheterized with the guidance of the 3DRA or 2D road map. Patients with the 3DRA road map had shorter total procedure time, catheterization time, fluoroscopy time, and smaller contrast medium volume and irradiation. Conclusions: the 3DRA road map is feasible and useful for the individualized guidance of UA catheterization and preferable to the conventional 2D road map.
\end{abstract}

Key words: Three-dimensional rotational angiography; Road mapping; Guidance; Uterine artery; Catheterization.

\section{Impact Statement}

- What is already known on this subject? Uterine artery (UA) embolization is an effective, minimally invasive, interventional procedure for treating gynaecological and obstetric diseases, such as symptomatic leiomyomas and postpartum haemorrhage. However, the process of catheterization is time-consuming because, sometimes, it is difficult to visualize UA origins under a conventional two-dimensional road map owing to vessels overlapping or foreshortening.

- What the results of this study add? Selection of optimal working projections from three-dimensional rotational angiography (3DRA) for identifying a UA origin and guiding catheterization individually can lead to radiation-dose and procedure-time reduction for cases requiring multiple oblique two-dimensional runs.

- What are the implications of these findings for clinical practice and/or further research? A 3DRA road map is feasible in pelvic region arterial interventions for gynaecological and obstetric diseases and also in cases of artery embolization for benign prostatic hyperplasia in males.

\section{Introduction}

Uterine artery (UA) embolization (UAE) is an effective, minimally invasive, interventional procedure for the treatment of symptomatic leiomyomas, postpartum haem- orrhage (PPH), uterine vascular malformations, pregnancy incisions, adenomyosis, and cervical cancer with bleeding. Sometimes, UAE can be considered as an alternative to myomectomy or hysterectomy $[1,2]$.

Catheterization of the UA is a prerequisite for a subsequent embolization treatment. This process is timeconsuming because, sometimes, it is difficult to visualize UA origins under conventional two-dimensional (2D) angiography owing to unappreciated vessels overlapping or foreshortening, resulting in an increase in the procedure time, contrast agent dose, and radiation dose. However, the procedure must be finished quickly and accurately during acute PPH; moreover, it is very important to keep the exposure as low as possible for patients who are young and willing to remain fertile.

Three-dimensional (3D) road maps and navigation techniques have shown their usefulness and potential capacity for time and dose reduction in interventional catheterdirected procedures for intracranial arteries [3, 4]. However, there are insufficient available clinical or radiation data regarding their use in UAE. Thus, we attempted to adopt this technique to guide the catheterization of the UA and compare its feasibility and advantages to those of conventional $2 \mathrm{D}$ road maps. 
Table 1. - Demographic data and uterine diseases.

\begin{tabular}{lccc}
\hline Characteristics & $2 \mathrm{D}\left(\mathrm{n}_{1}=10\right)$ & $3 \mathrm{D}\left(\mathrm{n}_{2}=10\right)$ & \\
\hline Age (years) & $43.7 \pm 3.6$ & $45.3 \pm 4.2$ & $P>0.05(\mathrm{u}=0.34)$ \\
Abdominal perimeter & $88.5 \pm 7.6$ & $83.2 \pm 9.1$ & $P>0.05(\mathrm{u}=1.41)$ \\
Symptomatic uterine fibroid & 4 & 4 & \\
Postpartum hemorrhage (PPH) & 2 & 3 & \\
Incision pregnancy & 2 & 1 & \\
Adenomyosis & 1 & 0 & \\
Cervical cancer & 1 & 2 & \\
\hline
\end{tabular}

Table 2. - Procedure time, UA catheterization time, fluoroscopy time, contrast volume, radiation dose.

\begin{tabular}{lccc}
\hline Characteristics & $2 \mathrm{D}\left(\mathrm{n}_{1}=10\right)$ & $3 \mathrm{D}\left(\mathrm{n}_{2}=10\right)$ & $P$ value \\
\hline Procedure time $(\mathrm{min})$ & $62.8 \pm 16.9$ & $45.0 \pm 14.8$ & $P<0.05(\mathrm{u}=2.50)$ \\
UA catheterization time $(\mathrm{min})$ & $13.7 \pm 5.4$ & $8.2 \pm 6.1$ & $P<0.05(\mathrm{u}=2.13)$ \\
Fluoroscopy time $(\mathrm{min})$ & $21.4 \pm 8.2$ & $13.5 \pm 7.6$ & $P<0.05(\mathrm{u}=2.23)$ \\
Total contrast volume $(\mathrm{mL})$ & $98.4 \pm 18.5$ & $70.7 \pm 15.9$ & $P<0.05(\mathrm{u}=3.58)$ \\
Radiation dose (DAP) $\left(\mathrm{Gy} . \mathrm{cm}^{2}\right)$ & $199.3 \pm 42.7$ & $160.4 \pm 35.2$ & $P<0.05(\mathrm{u}=2.22)$
\end{tabular}

DAP: dose area product.

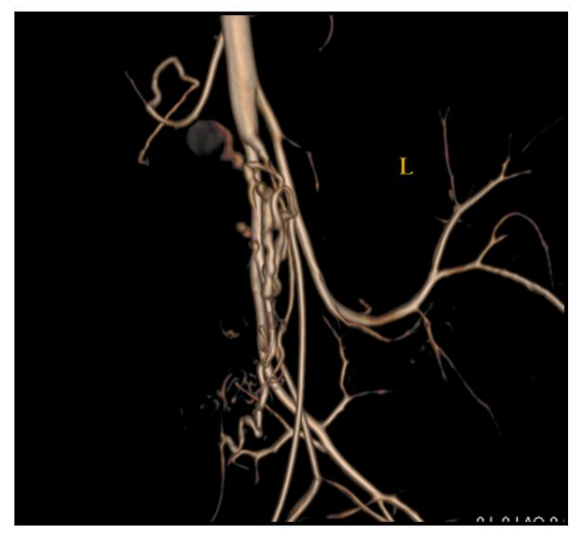

(a)

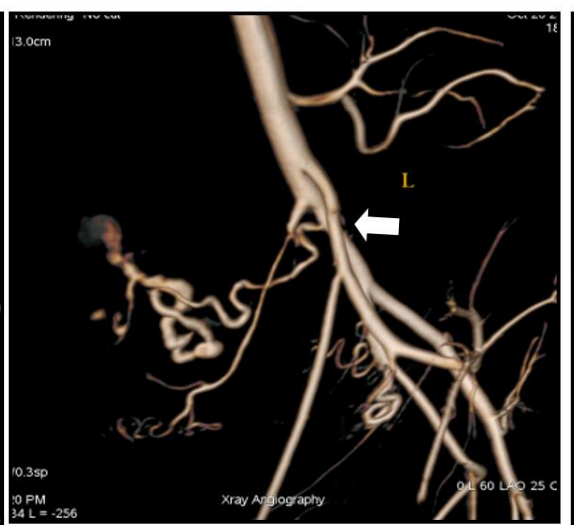

(b)

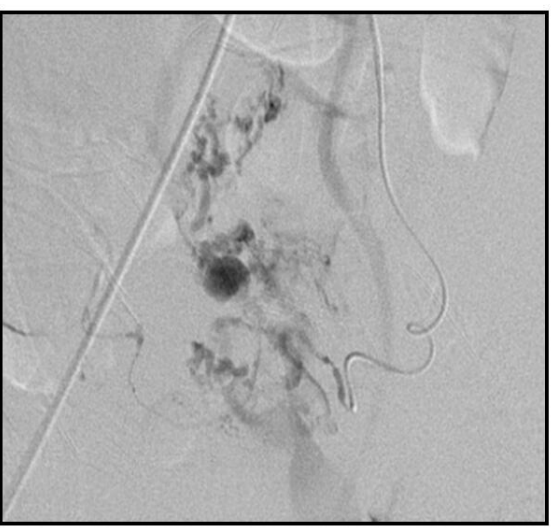

(c)

Figure 1. - A 38-year-old woman with postpartum hemorrhage was selected for uterine artery embolization. (a) The origin of left UA could not be visualized at posteroanterior for pressed displacement. (b) 3DRA showed the best view of the proximal left UA (arrows) was RAO $60^{\circ}$. (c) The selected projection angles was projected on the fluoroscopy screen as roadmap and the microcatheter was cannulated into the distal left UA accurately and quickly.

\section{Materials and Methods}

\section{Study design and patient population}

This prospective, randomized trial was conducted at our hospital by the Departments of Radiology and Gynecology. Ethical committee ([2018.08]NO.24) approval was provided for the Departments participating in this trial. Written informed consent was obtained from all patients after extensive counselling, by both the attending gynaecologist and interventionist. Twenty patients were randomly assigned to undergo UAE using a three-dimensional rotational angiography (3DRA) or conventional 2D road map in a ratio of 1 : 1 , with 10 cases in each group. Patient demographic data were analysed, including age, abdominal perimeter, and diseases. Group means of data were com- pared using unpaired U-tests. Demographic data are summarized in Table 1.

\section{Equipment and puncture procedure}

The following were used: GE Innova 3100 flat panel C-arm machine (Boston, Massachusetts, USA); amorphous flat panel detector and 3D reconstruction system; 3D workstation of Advantage Workstation 4.5 system; contrast medium (iopamidol, $300 \mathrm{mgI} / \mathrm{mL}$, Bayer HealthCare, Berlin, Germany); 5-French (F) vascular sheath, 5-F pigtail catheter, and 5-F Cobra catheter (Cook, USA); and a 2.7-F microcatheter (Terumo Europe, Leuven, Belgium).

The use of fluoroscopy (15 pulses/s) was minimized where possible, and the field of view was collimated to the central pelvis. All procedures were performed in a dedi- 


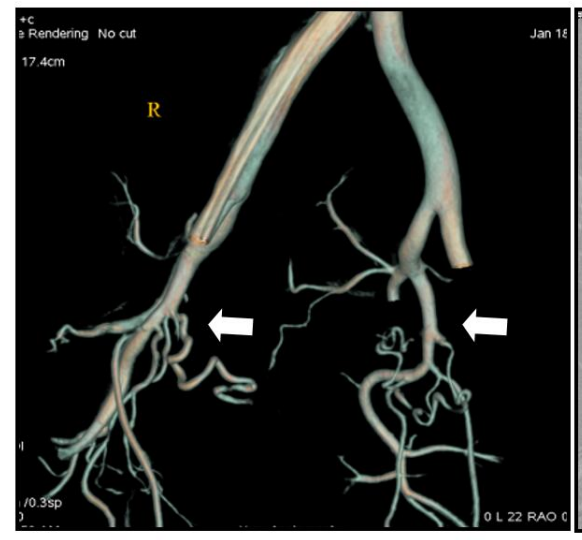

(a)

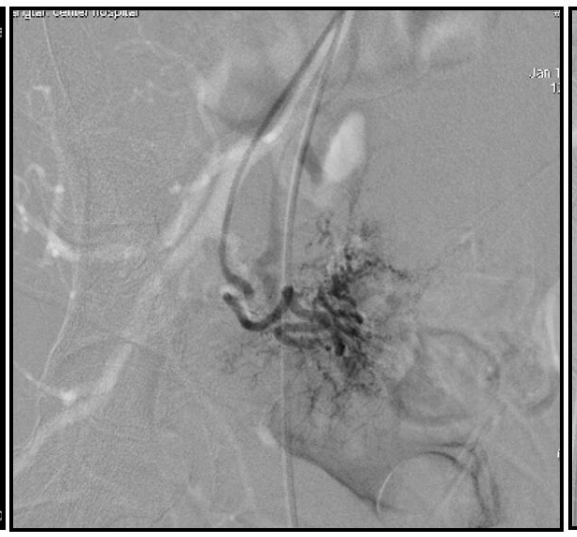

(b)

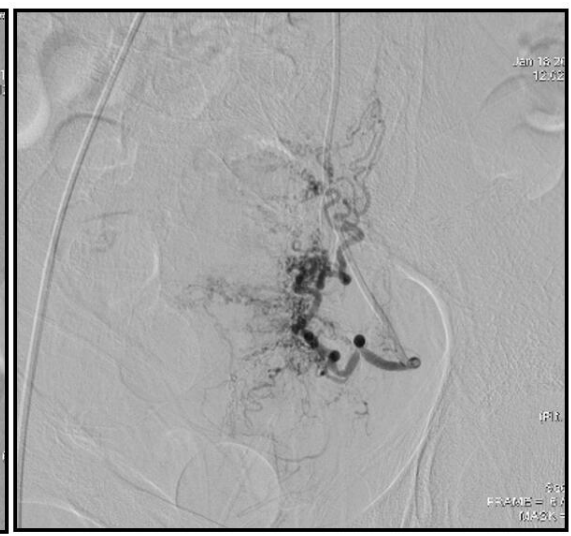

(c)

Figure 2. - A 45-year-old woman with symptomatic uterine fibroids was selected for uterine artery embolization. (a) 3DRA showed RAO $22^{\circ}$ was the optimal angiographic projection angle for identifying both sides of the UA origins. (b), (c) The C-arm was automatically steered to the corresponding angle, then catheterization of the two UAs were finished exactly and successfully guided by the roadmap.

cated angiosuite with a flat panel detector-equipped C-arm by the same interventional radiologist and technologist who both had $>10$ years of experience in UAE, with access to the right or left common femoral artery (single femoral approach).

\section{Acquisition of a 3DRA road map}

After obtaining access to the right common femoral artery using the 5-F vascular sheath, a 5-F pigtail catheter was placed in the distal abdominal aorta. Fluoroscopy was performed in the posterior-anterior, and lateral views, with the central pelvis as the area of interest. The TEST key was pressed. Then, the $\mathrm{C}$-arm automatically set the starting position at left anterior oblique (LAO) $102^{\circ}$ and the end position at right anterior oblique (RAO) $102^{\circ}$ with running a $204^{\circ}$ rotation to test. Then, a non-ionic iodinated contrast material (iopamidol, $300 \mathrm{mgI} / \mathrm{mL}$ ) was injected into the aorta and pelvic arteries through the pigtail catheter by an automatic pumping high-pressure syringe at a rate of 10 $\mathrm{mL} / \mathrm{s}$, volume of $50 \mathrm{~mL}$, and pressure of 300 psi during the $204^{\circ}$ rotation of the $\mathrm{C}$-arm with a speed of $40^{\circ} / \mathrm{s}$ and a total duration of $5.1 \mathrm{~s}$. The contrast injection began $2 \mathrm{~s}$ before $3 \mathrm{D}$ road map acquisition. A total of 90 images were acquired.

Imaging data from the rotation were then automatically transferred to Workstation 4.5 (GE, Boston, Massachusetts, USA). A 3D angiogram was reconstructed within seconds after acquisition. Working projections were expressed as degrees of angulation (caudal-cranial movement of the Carm) and rotation (LAO and RAO views). Using $3 \mathrm{D}$ visualization tools, the interventional radiologist could select the best view for catheterization of the UA (Figure 1 and Figure 2 ). The $\mathrm{C}$-arm was then automatically adjusted to the selected position, and the selected image was overlaid on the reference screen. After placing the 5-F Cobra catheter in the internal iliac artery and performing angiography again, the road map navigation for UA catheterization was obtained. Further, super-selective UA catheterization was performed using a coaxial microguidewire and microcatheter (Terumo Europe, Leuven, Belgium). Catheterization of the ipsilateral UA required forming a Waltman loop with the Cobra catheter.

\section{Acquisition of a $2 D$ road map}

After obtaining access to the right common femoral artery, the 5-F Cobra catheter was placed in the internal iliac artery. Through the Cobra catheter, "standard" $25^{\circ}$ ipsilateral or contralateral oblique angiography ( 3 frames/s) of the internal iliac artery and terminal branches was performed using $10 \mathrm{~mL}$ of a non-ionic iodinated contrast medium (iopamidol, $300 \mathrm{mgI} / \mathrm{mL}$ ) at an injection rate of $4 \mathrm{~mL} / \mathrm{s}$. When the origin of the UA was not clearly shown owing to the overlap of other vascular structures, an additional run was performed, with the obliquity adjusted at the operator's discretion and using the same frame and injection rates. Once the optimal angiographic view was obtained, an angiographic image was selected for projection overlay on the reference screen or to serve as a road map. Further, super-selective UA catheterization was performed using a coaxial microcatheter (Terumo Europe, Leuven, Belgium). Catheterization of the ipsilateral UA required forming a Waltman loop with the Cobra catheter.

\section{Embolization procedure}

Embolization was performed through a microcatheter after confirmatory Digital Substraction Angiography (DSA) by an automated pump injector $(3 \mathrm{~mL} / \mathrm{s}$, total of $8 \mathrm{~mL})$. All embolization procedures were performed under fluoroscopic guidance through a microcatheter in the posteroanterior view using 500-700- $\mu \mathrm{m}$ and 700-900- $\mu \mathrm{m}$ microspheres (Embosphere, Biosphere Medical, USA) mixed with iopamidol, until stasis was achieved.

All procedures were performed bilaterally. Angiography of the internal iliac artery was performed through the 5 -F Cobra catheter $(4 \mathrm{~mL} / \mathrm{s}$, total of $10 \mathrm{~mL}$ ) after complet- 
ing each side UAE procedure. After bilateral embolization, a DSA aortogram (20-mL contrast medium) was obtained to exclude ovarian artery supply to the fibroid tumours or haemorrhage.

\section{Statistical analysis}

Several parameters were recorded sequentially, including procedure time, UA catheterization time, fluoroscopy time, total contrast agent volume, and radiation dose. Procedure time was defined as the time from femoral access to vascular closure. Catheterization time of the left UA was defined as the time from right femoral puncture to catheterizing a microcatheter into the left UA and catheterization time of the right UA was defined as the time from completion of left UAE to catheterizing a microcatheter into the right UA

Fluoroscopy time, total contrast agent volume, and radiation dose were cumulatively recorded at various stages of the procedure from femoral access to completion of bilateral embolization.

Numeric variables were presented as mean \pm standard deviation and $95 \%$ confidence interval. The date between 3DRA and 2D groups were compared using the unpaired Mann-Whitney U-test. One-sided $P$ values were considered significant if $<0.05$.

\section{Results}

All 20 patients (40 UAs) successfully underwent UA catheterization and embolization in the two groups. No ovarian artery was catheterized and embolized. There was no statistical difference in the demographic characteristics between the two groups (Table 1).

For all 10 patients in the 3DRA group, it was technically feasible to perform 3D rotational angiography of both internal iliac arteries and select one optimal obliquity to perform super-selective catheterization of the UA. In the 2D group, a mean of 2.8 (range from 2 to 4 ) exposures was performed to obtain the best view of the origin of the UA for the subsequent super-selective catheterization.

Procedural parameters were summarized in Table 2 . These data clearly demonstrated that compared with patients in the 2D group, patients in the 3D group had shorter total procedure time, catheterization time, and fluoroscopy time, as well as smaller contrast medium volume and irradiation $(P<0.05$ for all). There were significant differences between the two groups.

\section{Discussion}

Since UAE was first described by Ravina et al. in 1995, it has been shown to be a safe, effective, and cost-effective alternative to traditional surgical options, with data from long-term studies currently available [5]. It is widely accepted that UAE is an effective therapeutic strategy for symptomatic uterine fibroids and $\mathrm{PPH}$ of various causes along with conventional surgical treatment of hysterectomy and myomectomy. It has the advantage of being fast and repeatable and can be performed without general or lumbar anaesthesia. Moreover, it preserves the uterus and makes future menstruation and fertility possible [6-8]

Accurate and rapid catheterization of the UA is the key to the procedure and a prerequisite for subsequent embolization. However, catheterization of the UA often takes most of the procedure time because of a significant anatomic variability in the origins of the UAs and vessels overlapping. Other factors include the diameter of the UA, pressed displacement, the use of haemostasis drugs, the age of patients, and the interventionist's experience. Thus, visualizing the UA origin clearly and inserting a microcatheter in the UA within a short time without complications, such as arterial spasm, dissection, or perforation, are very important. Accurate and rapid catheterization of the UA is not just for as low as possible exposure for patients who are young and potentially willing to remain fertile, but also for the condition of acute PPH.

The current and established technique (2D) of UA catheterization relies on the interventionist choosing a somewhat arbitrary starting projection or angle, with subsequent pelvic DSA. The initially selected projection angles may not be optimal. The interventionist could spend a significant amount of time searching for the origin of the UA and using several different obliquity angles before catheterizing successfully in the conventional 2D technique [9]. This would result in an increase in procedure time, contrast agent dose, radiation dose, and risk of vascular injury, spasm, and haemorrhage [10].

3DRA is a procedure in which the $\mathrm{C}$-arm rotates automatically at a constant speed during angiography when a catheter placed in the artery of the interest. Then, the 3D reconstruction image of the pelvic arterial tree can be obtained. The selected image and angle of the $\mathrm{C}$-arm can serve as a road map for the navigation of UA catheterization, overcoming the limitations of 2D imaging, owing to overlying vessels. 3DRA has been popularly used in cerebral angiography to identify subtle lesions and guide the embolization treatment of cerebral aneurysm under the road map generated from 3DRA scans [11-13]. Studies in neuroradiology have shown that the pretreatment selection of optimal working projections from 3DRA can lead to radiation dose reduction for cases requiring multiple oblique $2 \mathrm{D}$ runs [14-16].

Thus, we theorized that 3DRA seemed to be feasible in pelvic region arterial interventions such as in the intracranial arteries without cardiac and respiratory movements in the upper abdomen and thorax. There had been a few studies about 3DRA being used in UA angiography, but the main goals of the procedures were limited to exploring and summarizing a standard projection angle or a range of angles, which could identify the UA origin and consistently be utilized for UA catheterization in 2D angiography [17]. The conclusions were widespread different from each other. Some authors have reported that angiography performed at the contralateral oblique angle of $31^{\circ}-45^{\circ}$ was sufficient to 
visualize the origin of the $\mathrm{UA}$, but some reported $\mathrm{LAO}$ angles of $20^{\circ}-50^{\circ}$ or RAO angles of $20^{\circ}-50^{\circ}$ and others reported homolateral oblique angles of $31^{\circ}-45^{\circ}$ [18-20]. The caudal-cranial range was smaller with angulations, between Cran $10^{\circ}$ and Caud $10^{\circ}$. In fact, there are no standard projection angles or fixed range recommended to clearly visualize all UA origins for individual variations. Relying on a projection of an LAO angle of $45^{\circ}$ or RAO angle of $45^{\circ}$ appears to clearly identify the UA origin only $50 \%$ of the time, which is inadequate for accurate and rapid catheterization [21].

In this study, the total procedure time and fluoroscopy time in the 3DRA group were shorter than those in the 2D group because the cannulation time was significantly shortened and 3DRA acquisition time was very short $(5.1 \mathrm{~s})$. The total procedure time and fluoroscopy time were also preferable to other recent reports, in which they were 63-96 minutes and 16.6-29.5 minutes. Enhanced techniques described to reduce the procedure time include preselecting projection angles from preprocedure Computed Tomography Angiography (CTA) or Magnetic Resonance Angiography (MRA) (51.9 minutes) or bilateral femoral artery punctures and simultaneous bilateral embolization (54.9 minutes) [22, 23]. A live MRA road map overlay (45.3 minutes) was even faster than above enhanced techniques in guidance of UA catheterization [24], but it needed time to acquire an MRA or a CTA before the procedure, which increased the total procedure time. Moreover, the learning curve for both the interventionist and technologist performing the overlay was long. The total procedure time of using 3DRA was shorter than that of MRA and live MRA road map owing to no need of preprocedur MR. Additionally, 3DRA was a very helpful technique for acute PPH.

We performed 3DRA and acquired images of both sides of the internal iliac arteries and UA origins together by placing a 5-F pigtail catheter in the distal abdominal aorta, instead of performing rotational angiography for each internal iliac artery. The total procedure time, radiation dose, and contrast agent dose were further reduced compared to those in the study by Geert et al. [25].

Our study demonstrated that 3DRA and 3D road mapping were feasible and accurate for individualized catheter guidance during UAE. Interventionists with less experience in performing the treatment were also able to find a suitable viewing angle of the UAs from 3DRA and successfully perform catheterization. No additional complications of UAE had been found when using a $3 \mathrm{D}$ road map compared with using a 2D road map, although complications were not in the scope of this discussion. The main goal of our study was not to evaluate the whole UA but to localize the proximal part of the UA and select optimal projection angles for further individualized cannulation.

A limitation of this study was the low number of cases and different diseases involved, but our preliminary experience showed that the efficiency of the procedure had improved greatly without increasing the cost and risk. There- fore, we considered that 3DRA was a good selection for individualized guidance catheterization of UAs owing to its characteristics of fast pace, excellent effect. It can be popularized in clinics in the future.

\section{Acknowledgments}

I would like to express my gratitude to all those who helped me during the writing of this manuscript. Thanks to all the peer reviewers and editors for their opinions and suggestions.

\section{Conflict of Interest}

The authors declare no competing interests.

Submitted: October 23, 2019

Accepted: April 21, 2020

Published: October 15, 2020

\section{References}

[1] Hehenkamp W.J., Volkers N.A., Biernie E., et al.: "Symptomatic uterine fibroids, treatment with uterine artery embolization or hysterectomy, results from the randomized clinical Embolization versus Hysterectomy (EMMY) trial". Radiology, 2008, 246, 823-832.

[2] Edwards R.D., Moss J.G., Lumsden M.A., et al.: "Committee of the randomized trial of embolization versus surgical treatment for fibroids". N. Engl. J. Med., 2007, 356, 360-370.

[3] Soderman M., Babic D., Homan R., et al.: "3D roadmap in neuroangiography, technique and clinical interest". Neuroradiology, 2005, 47, 735-740.

[4] Liapi E., Hong K., Georgades C.S., et al.: "Three dimensional rotational angiography, introduction of an adjunctive tool for successful transarterial chemoembolization". J. Vasc. Intervent. Radiol., 2005, 16, 1241-1245.

[5] Ravina J.H., Herbreteau D., Ciraru-Vigneron N., et al.: “Arterial embolisation to treat uterine myomata". Lancet, 1995, 346, 671-672.

[6] Gonsalves M., Belli A.: "The role of interventional radiology in obstetric hemorrhage". Cardiovasc. Intervent. Radiol., 2010, 33, 887895.

[7] Cristina M., Yen Z.,Anju S., et al.: "Preprocedural MRI and MRA in planning fibroid embolization”. Diagn. Interv. Radiol., 2017, 23, 163-171.

[8] Cheng S., Sang M., Jong W., et al.: "Recent Update of Embolization of Postpartum Hemorrhage". Korean J. Radiol., 2018, 19, 585-596.

[9] Pelage J.P., Wassef M., Lacombe P., Laurent A.: "Technique of uterine artery embolization and choice of embolics". Philadelphia: Lippincott Williams and Wilkins, 2004, 82-94.

[10] Naguib N.N., Nour-Eldin N.E., Lehnert T., et al.: "Uterine artery embolization, optimization with preprocedural prediction of the best tube angle obliquity by using 3D-reconstructed contrast-enhanced MR angiography". Radiology, 2009, 251, 788-795.

[11] Wong SC., Nawawi O., Ramli N., Abd Kadir K.A.: "Benefits of $3 \mathrm{D}$ rotational DSA compared with 2D DSA in the evaluation of intracranial aneurysm". Acad. Radiol., 2012, 19, 701-707.

[12] Soderman M., Babic D., Homan R., et al.: "3D roadmap in neuroangiography, technique and clinical interest”. Neuroradiology, 2005, 47, 735-740.

[13] Tsapaki V., Vano E., Muavrikou I., et al.: "Comparison of patient dose in two-dimensional carotid angiography and three-dimensional rotational angiography". Cardiovasc. Intervent. Radiol., 2008, 31, 477-482.

[14] Schueler B.A., Kallmes D.F., Cloft H.J.: “3D cerebral angiography, radiation dose comparison with digital subtraction angiography".Am. J. Neuroradiol., 2005, 26, 1898-1901.

[15] Wong S.C., Nawawi O., Ramli N., Abd Kadir K.A.: "Benefits of $3 \mathrm{D}$ rotational DSA compared with 2D DSA in the evaluation of intracranial aneurysm". Acad. Radiol., 2012, 19, 701-707.

[16] Gupta A., Radaelli A.G.: "Live 3D guidance in endovascular procedures". Endovascular Today, 2009, 7, 28-40. 
[17] Shen Z.., Han J., Hu D., et al.: "Uterine artery opening photography angle analysis". J. Interv. Radiol., 2016, 25, 116-119.

[18] Jian S., Ran B.: "The ratio of uterine artery opening position in angiography of internal iliac arteries with different projection angles". Journal of Jiangxi Medical College, 2003, 43, 106-108.

[19] Yang J., He N., Zhang C., et al.: "Pelvic arteriography anteroposterior and oblique position on uterine artery opening comparisons shown".Clin. Radiol., 2003, 22, 324-326.

[20] Lv W., Zhang A., Zhou C., et al.: "Three-dimensional DSA manifestation of uterine artery and its best display positional discussion". J. Interv. Radiol., 2011, 20, 853-856.

[21] Atul G., Kirsten Z., Thijs G., et al.: "Three-dimensional rotational angiography is preferable to conventional two-dimensional techniques for uterine artery Embolization". Diagn. Interv. Radiol., $2013,19,418-426$.

[22] Mori K., SaidaT., ShibuyaY., et al.: "Assessment of uterine and ovarian arteries before uterine artery embolization, advantages conferred by unenhanced MR angiography". Radiology, 2010, 255, 467-475.

[23] Costantino M., Lee J., McCullough M., et al.: "Bilateral versus uni- lateral femoral access for uterine artery embolization, results of a randomized comparative trial". J. Vasc. Interv. Radiol., 2010, 21, 829-835.

[24] Atul G., Thijs G.: "Live MR angiographic roadmapping for uterine artery embolization, a feasibility study". J. Vasc. Interv. Radiol., 2013, 24, 1690-1697.

[25] Geert M., Koen M., Dirk T., et al.: "2D versus 3D roadmap for uterine artery catheterization, impact on several angiographic parameters". Acta. Radiologica, 2014, 55, 62-70.

\section{Corresponding Author:}

WENQUAN LI, M.D.

Department of Interventional Radiology

Affiliated Hospital of Hunan institute of traditional Chinese medicine

Changsha 410013

Hunan (P.R. China)

e-mail: liwenquan0000@163.com 